\title{
Common Bacterial Infections and Risk of Dementia or Cognitive Decline: A Systematic Review
}

\author{
Rutendo Muzambi $^{\mathrm{a}, *}$, Krishnan Bhaskaran ${ }^{\mathrm{a}}$, Carol Brayne ${ }^{\mathrm{b}}$, Jennifer A. Davidson ${ }^{\mathrm{a}}$, \\ Liam Smeeth ${ }^{\mathrm{a}}$ and Charlotte Warren-Gash ${ }^{\mathrm{a}}$ \\ ${ }^{a}$ Faculty of Epidemiology and Population Health, London School of Hygiene and Tropical Medicine, \\ London, UK \\ ${ }^{\mathrm{b}}$ Cambridge Institute of Public Health, Cambridge University, Cambridge, UK
}

Accepted 29 May 2020

\begin{abstract}
.
Background: Bacterial infections may be associated with dementia, but the temporality of any relationship remains unclear. Objectives: To summarize existing literature on the association between common bacterial infections and the risk of dementia and cognitive decline in longitudinal studies.

Methods: We performed a comprehensive search of 10 databases of published and grey literature from inception to 18 March 2019 using search terms for common bacterial infections, dementia, cognitive decline, and longitudinal study designs. Two reviewers independently performed the study selection, data extraction, risk of bias and overall quality assessment. Data were summarized through a narrative synthesis as high heterogeneity precluded a meta-analysis.

Results: We identified 3,488 studies. 9 met the eligibility criteria; 6 were conducted in the United States and 3 in Taiwan. 7 studies reported on dementia and 2 investigated cognitive decline. Multiple infections were assessed in two studies. All studies found sepsis $(n=6)$, pneumonia $(n=3)$, urinary tract infection $(n=1)$, and cellulitis $(n=1)$ increased dementia risk (HR 1.10; 95\% CI 1.02-1.19) to (OR 2.60; 95\% CI 1.84-3.66). The range of effect estimates was similar when limited to three studies with no domains at high risk of bias. However, the overall quality of evidence was rated very low. Studies on cognitive decline found no association with infection but had low power.

Conclusion: Our review suggests common bacterial infections may be associated with an increased risk of subsequent dementia, after adjustment for multiple confounders, but further high-quality, large-scale longitudinal studies, across different healthcare settings, are recommended to further explore this association.
\end{abstract}

Keywords: Cognition, dementia, infections, prevention, systematic review

Systematic review registration number: CRD42018119294, registered in December 2018

\section{INTRODUCTION}

Dementia is a major global health challenge. Worldwide, approximately 50 million people are

\footnotetext{
*Correspondence to: Rutendo Muzambi, Faculty of Epidemiology and Population Health, London School of Hygiene and Tropical Medicine, London, WC1E 7HT, UK. Tel.: +44 0207299 4678; E-mail: rutendo.muzambi@1shtm.ac.uk.
}

currently living with dementia, and this number is projected to rise to over 152 million by 2050 [1]. Given the increasing life expectancy and absence of a cure or disease-modifying therapy, dementia prevention has become a public health priority [2]. Recent evidence suggests modifiable risk factors may have contributed to a decline in the age-specific incidence of dementia in Europe and the United 
States [3-9], highlighting the importance of identifying and targeting modifiable risk factors, as age increases. Bacterial infections have been identified as one potentially important risk factor for dementia $[10,11]$.

Symptomatic bacterial infections such as pneumonia and urinary tract infections are common, and complications frequently occur among older people. One of the hallmark complications of common bacterial infections is delirium; a serious neuropsychiatric syndrome characterized by acute cognitive dysfunction and inattention [12]. Delirium is strongly associated with an increased risk of subsequent cognitive decline and dementia [13-15]. Increasing evidence suggests cognitive impairment may persist for years after sepsis hospitalization [16, 17]. However, it is unclear whether there are long term effects of common infections on cognition and dementia, independent of delirium.

Previous reviews have investigated the role of bacterial pathogens on Alzheimer's disease; however, evidence is inconsistent, and the exact nature of this association remains unclear. In a meta-analysis of predominantly case-control studies Maheshwari and Eslick found that Chlamydia pneumonia, a bacterium responsible for pneumonia and other respiratory tract infections, was associated with a five-fold (OR 5.66; 95\% CI 1.83-17.51) increased occurrence of Alzheimer's disease [11]. However, due to the crosssectional nature of the data collected in these studies, it was not possible to assess temporality. Additional drawbacks of this meta-analysis included differences in methodology and the relatively small sample sizes of the studies included (total sample size ranging from 2 to 200 samples). Furthermore, other bacterial microorganisms have also been implicated in previous and subsequent reviews [10, 18] and differing conclusions have been drawn on the role of Chlamydia pneumonia with Alzheimer's disease as evidenced in a comprehensive review by Mawanda and Wallace [18]. However, studies included in these reviews also faced the same limitations in terms of sample size and cross-sectional study designs.

We aimed to summarize current evidence from longitudinal studies of the association between common clinically symptomatic bacterial infections (sepsis, lower respiratory tract infections, urinary tract infections, and skin and soft tissue infections) and risk of subsequent incident dementia or cognitive decline in adults aged 18 years and older. A secondary objective was to identify gaps in literature and recommendations for future research.

\section{METHODS}

\section{Protocol and registration}

We registered this systematic review with the International Prospective Register of Systematic Reviews in December 2018 (PROSPERO 2018; CRD42018119294) and published a more detailed protocol in accordance with the Preferred Reporting Items for Systematic Reviews and Meta Analyses Protocols (PRISMA) reporting guidelines [19].

\section{Study design}

Studies eligible for inclusion were longitudinal studies such as prospective and retrospective cohort studies, secondary analyses of randomized controlled trial data, and case control studies. We included studies in which cognitive decline was measured at least 3 months following infection, to avoid associations dominated by short term, reversible cognitive impairment. Further, to assess temporality, we restricted our search to studies in which infections occurred prior to cognitive decline or dementia.

\section{Study population}

Only studies with human participants aged 18 years and older were eligible for inclusion.

\section{Exposure}

Exposure was defined as symptomatic illness due to common bacterial infections, either suspected clinically or confirmed by isolation of a bacterial pathogen. We identified studies investigating the following major infection types: sepsis, lower respiratory tract, skin and soft tissue, and urinary tract infections. Studies identifying specific bacterial agents alone, rather than the symptomatic disease, were excluded.

\section{Comparators}

We only included studies in which a comparison group was present. This comparison group comprised of individuals unexposed to common bacterial infections in cohort studies and secondary analyses of longitudinal randomized controlled trial data, or a control group without dementia or cognitive decline in case control studies. 


\section{Outcomes}

Our two primary outcomes of interest were (1) incident dementia (all types) and (2) cognitive decline. We included studies in which our outcomes were defined clinically, which for dementia could be with or without neuroimaging or histopathology results.

\section{Information sources}

We performed a comprehensive search across eight databases of published literature (MEDLINE, EMBASE, Global Health, PsychInfo, CINAHL Plus, Cochrane Library, Scopus, and Web of Science) and two grey literature databases (Open grey and the British Library electronic Theses Online Service) from inception to 18 March 2019. Additionally, we searched the reference lists of the included studies to identify any relevant articles not captured in our search strategy.

\section{Search}

We searched the databases using subject headings, specific to each database, and keywords related to common bacterial infections, cognitive decline or dementia, and longitudinal study designs. These search terms were then combined using Boolean logical operators. No restrictions were placed on the language, country, or health care setting of the studies. Our search strategy was developed in consultation with a librarian at the London School of Hygiene and Tropical Medicine and was subsequently peer reviewed based on the Peer-Review for Electronic Search Strategies. We translated the final search strategy across all databases which is shown in the Supplementary Material 1.

\section{Study selection}

The study selection process was carried out by two reviewers (RM and JAD), using the Preferred Reporting Items for Systematic Reviews and Meta-Analyses flow diagram. The two reviewers independently screened all titles, abstracts and full text articles against the eligibility criteria. A third reviewer (CWG) was consulted when there were any discrepancies.

\section{Data items}

Data extraction was performed independently by two reviewers for all studies. We used the Population, Exposure, Comparator, Outcomes and Study characteristics framework to extract data from eligible studies (Supplementary Material 2). We pilot tested our data extraction form and modified the form accordingly. We extracted key study results, namely unadjusted and adjusted incidence effect estimates and their corresponding 95\% CIs. Data on confounding variables adjusted for in each study and pre-specified sub-groups were also extracted.

\section{Risk of bias in individual studies}

The risk of bias was assessed independently by two reviewers in line with the Cochrane collaboration approach. We classified studies as at high, medium, low or unclear risk of bias in each of the following domains: confounding, selection of participants, misclassification of variables, missing data, reverse causation, generalizability, and study power [20, 21].

\section{Synthesis of results}

We grouped studies according to their outcome (cognitive decline or dementia) and exposure definition (common bacterial infection) and synthesized them narratively. Heterogeneity was assessed using the I squared statistic if there were two or more studies with effect estimates for the same exposure definition, outcome, and study design. Geographical location and risk of bias were explored as potential sources of heterogeneity. Other sources of heterogeneity could not be explored due to the limited data available. We also carried out sub-group analyses by age, sex, and dementia subtype where sufficient data were available.

\section{Quality of evidence}

We assessed the overall quality of evidence for each outcome using the Grading of Recommendations, Assessment, Development and Evaluation (GRADE) tool. The following domains were assessed: study limitations, inconsistency, indirectness, imprecision, and publication bias. We rated the strength of evidence as high, moderate, low, or very low. The criteria for grading are stated in Supplementary Material 3. 


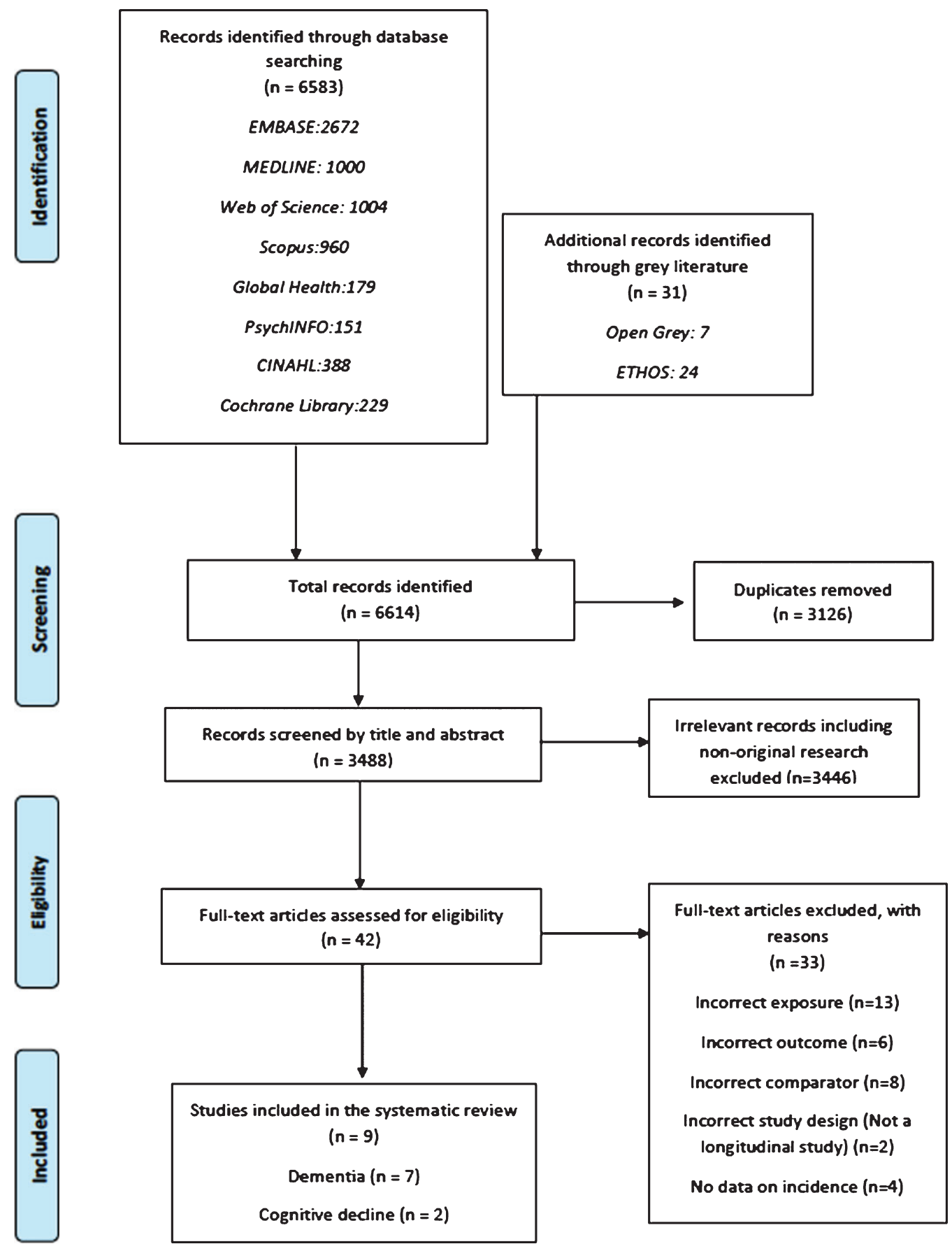

Fig. 1. Study selection PRISMA flow diagram.

\section{RESULTS}

\section{Study characteristics}

In total, 3,488 studies were identified from 10 databases, after de-duplication, as outlined in Fig. 1.
Of these, 42 were included in the full text screening and the reasons for exclusion were noted. Finally, 9 studies were included in the present systematic review. The study characteristics and results are summarized in Tables 1 and 2, respectively. Forest plots of the results are displayed in Figs. 2 and 3. 


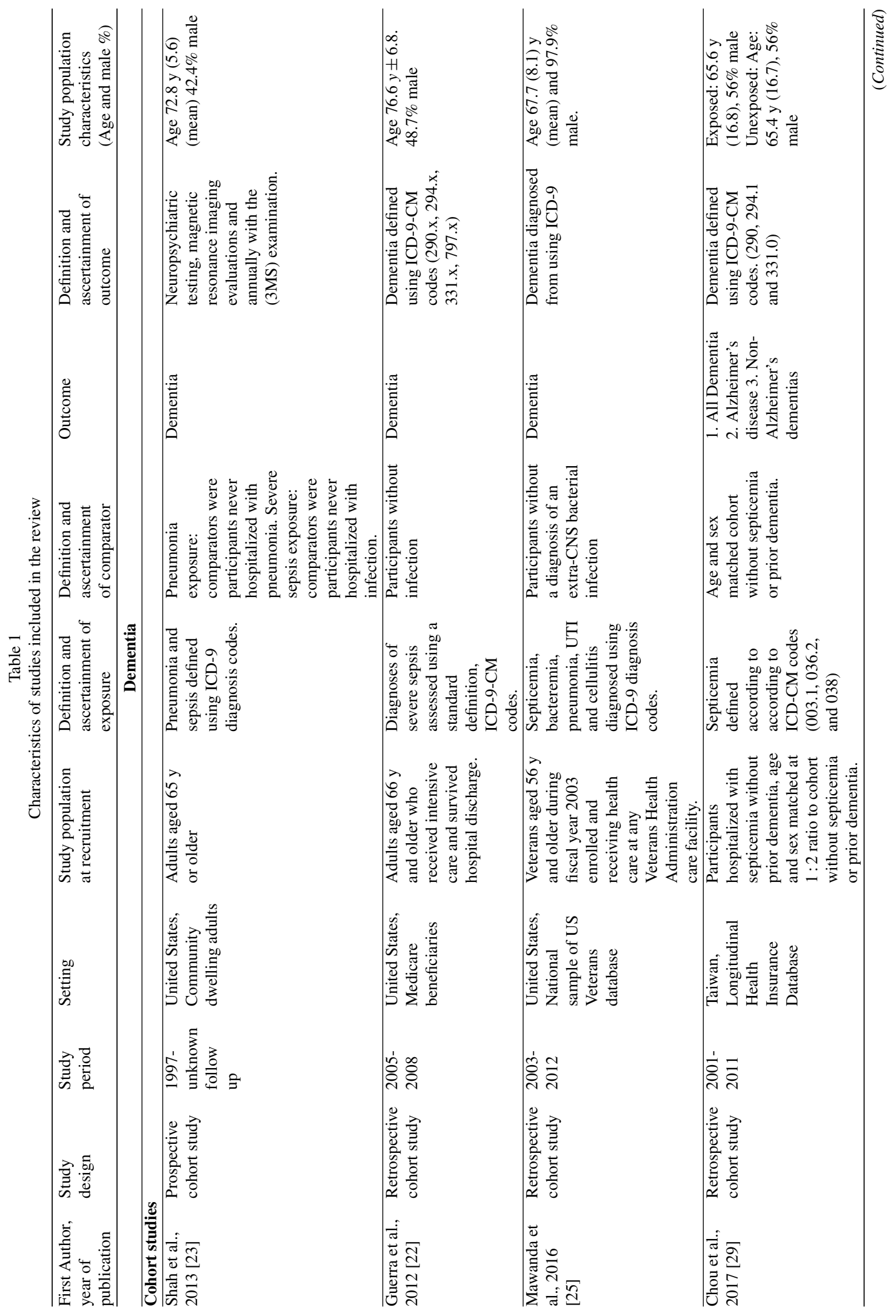




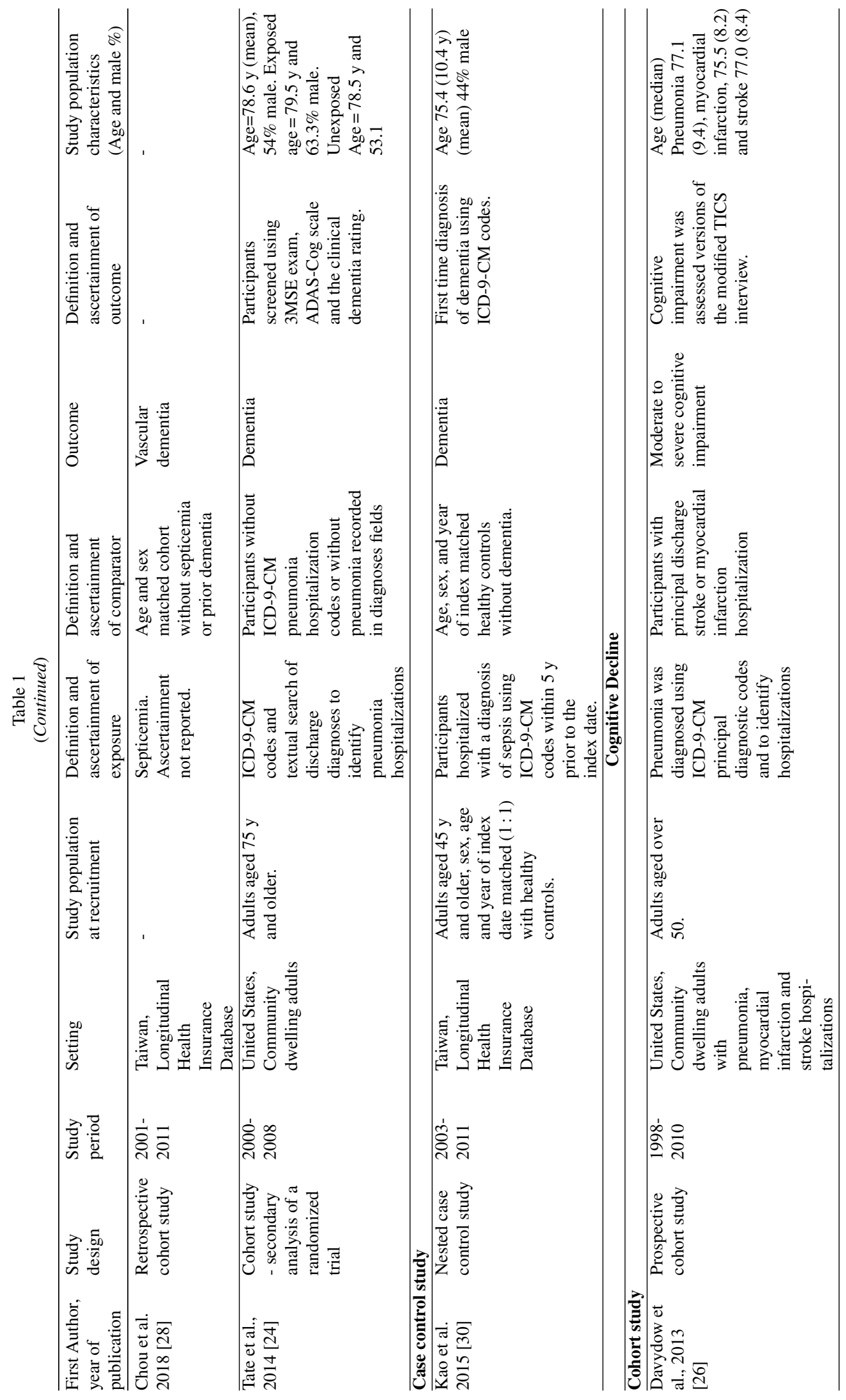




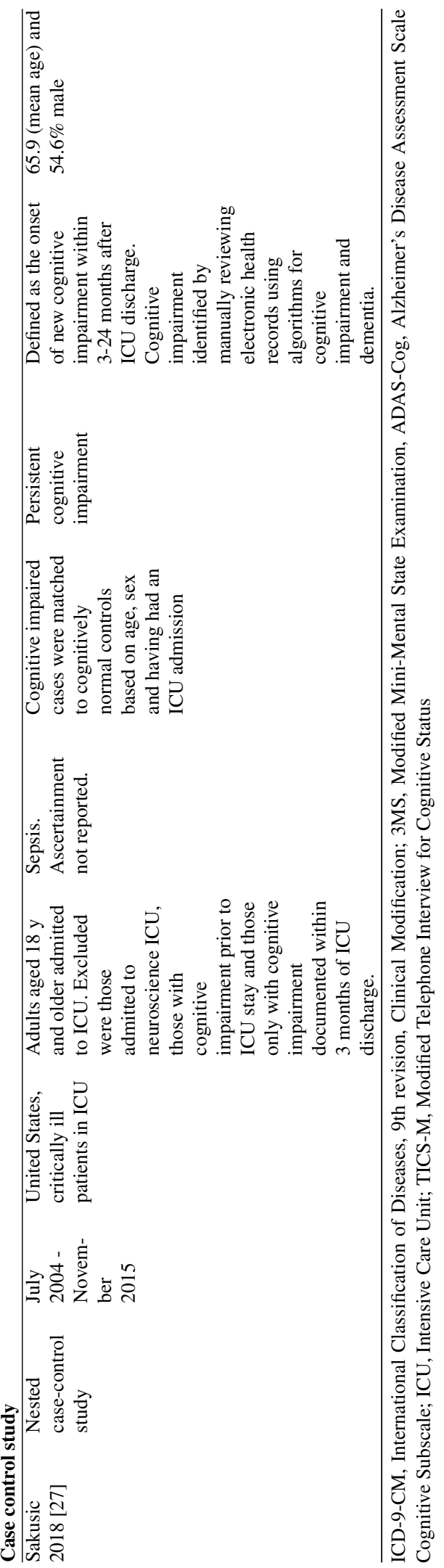




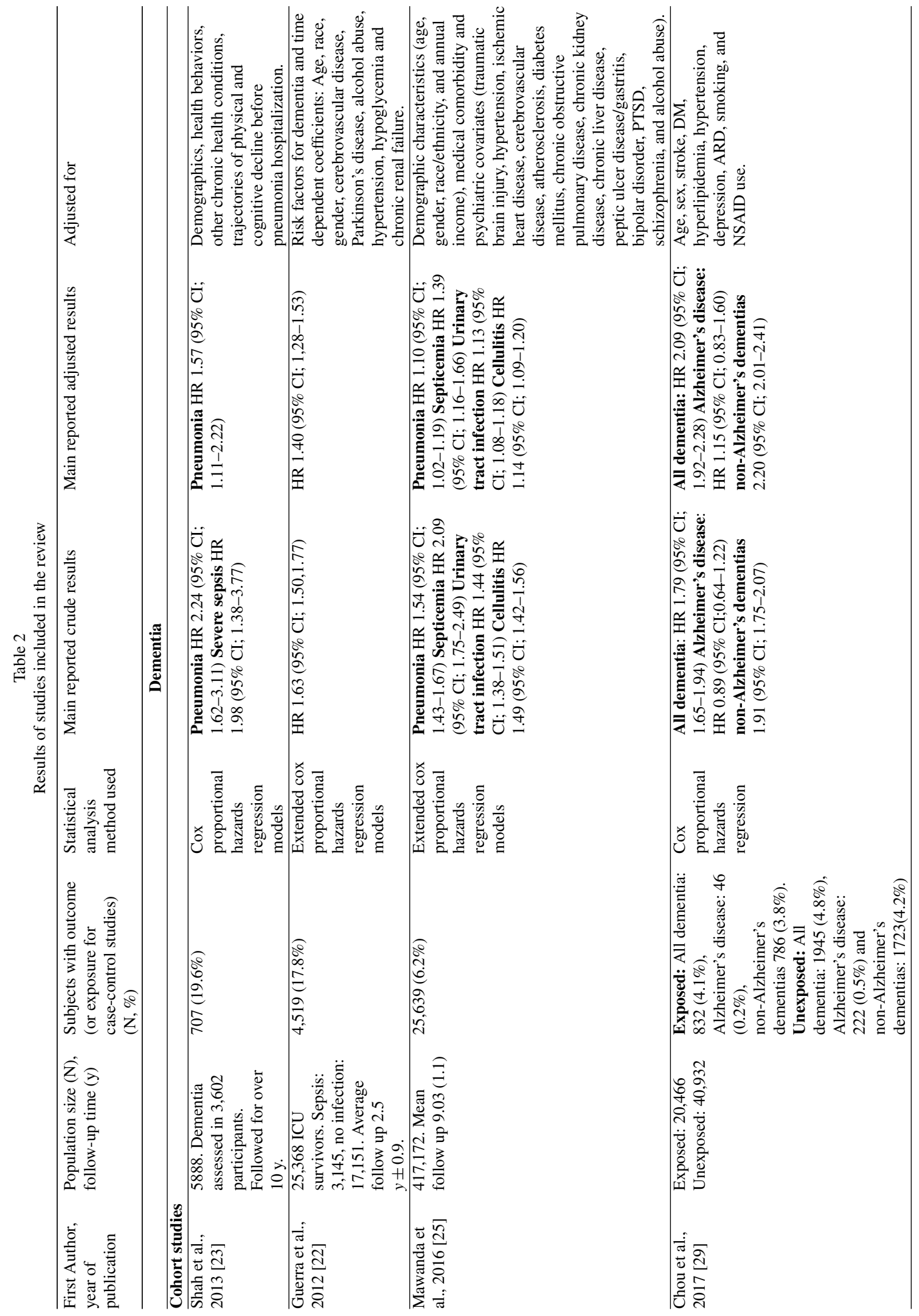




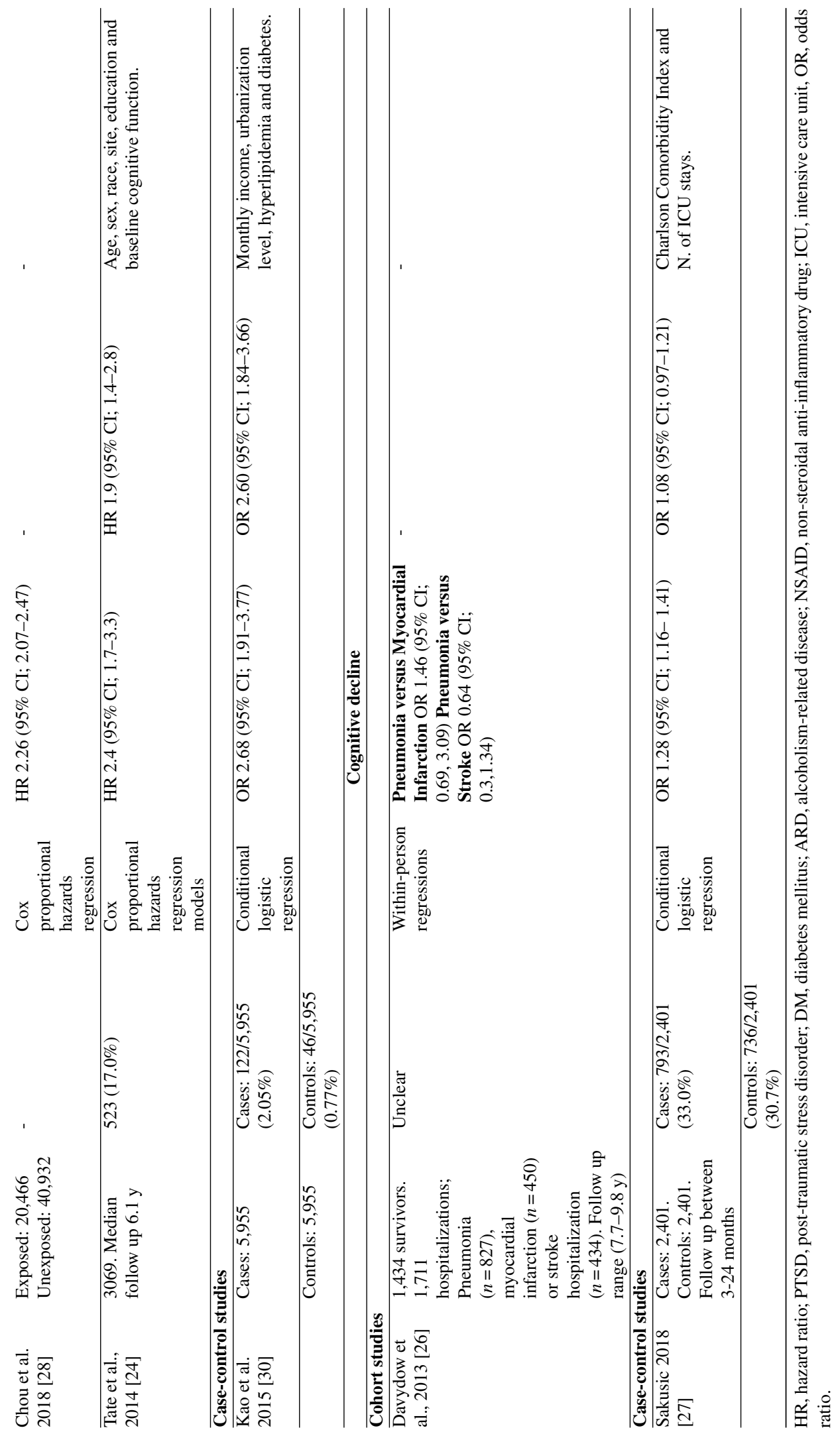




\begin{tabular}{|c|c|c|c|c|c|}
\hline \multirow[b]{2}{*}{ Author } & \multirow[b]{2}{*}{ Year } & \multirow[b]{2}{*}{ Country } & \multicolumn{3}{|l|}{ Effect } \\
\hline & & & Estimate & & ES $(95 \% \mathrm{Cl})$ \\
\hline \multicolumn{6}{|l|}{ Sepsis } \\
\hline Guerra et al & 2012 & USA & $H R$ & $\leftarrow$ & $1.40(1.28,1.53)$ \\
\hline Mawanda et al & 2016 & USA & $\mathrm{HR}$ & $\rightarrow$ & $1.39(1.16,1.66)$ \\
\hline Chou et al & 2017 & Taiwan & $H R$ & $\leftarrow$ & $2.09(1.92,2.28)$ \\
\hline Chou et al & 2018 & Taiwan & $H R$ & 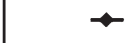 & $2.26(2.07,2.47)$ \\
\hline Kao et al & 2015 & Taiwan & OR & & $2.60(1.84,3.66)$ \\
\hline Shah et al & 2013 & USA & $H R^{*}$ & & $2.28(1.38,3.77)$ \\
\hline \multicolumn{6}{|l|}{ Pneumonia } \\
\hline Shah et al & 2013 & USA & $\mathrm{HR}$ & $\rightarrow$ & $1.57(1.11,2.22)$ \\
\hline Tate et al & 2014 & USA & $\mathrm{HR}$ & $\longrightarrow$ & $1.90(1.40,2.80)$ \\
\hline Mawanda et al & 2016 & USA & $\mathrm{HR}$ & 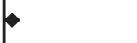 & $1.10(1.02,1.19)$ \\
\hline \multicolumn{6}{|l|}{ UTI } \\
\hline Mawanda et al & 2016 & USA & $H R$ & $\bullet$ & $1.13(1.08,1.18)$ \\
\hline \multicolumn{6}{|l|}{ Cellulitis } \\
\hline Mawanda et al & 2016 & USA & $H R$ & $\bullet$ & $1.14(1.09,1.20)$ \\
\hline
\end{tabular}

Fig. 2. Forest plot showing the effect of infections on dementia. *Unadjusted effect estimates. The mean age (SD) in this study was 72.8 years (5.6).

Effect
Author
Year Estimate Comparator
ES $(95 \% \mathrm{Cl})$

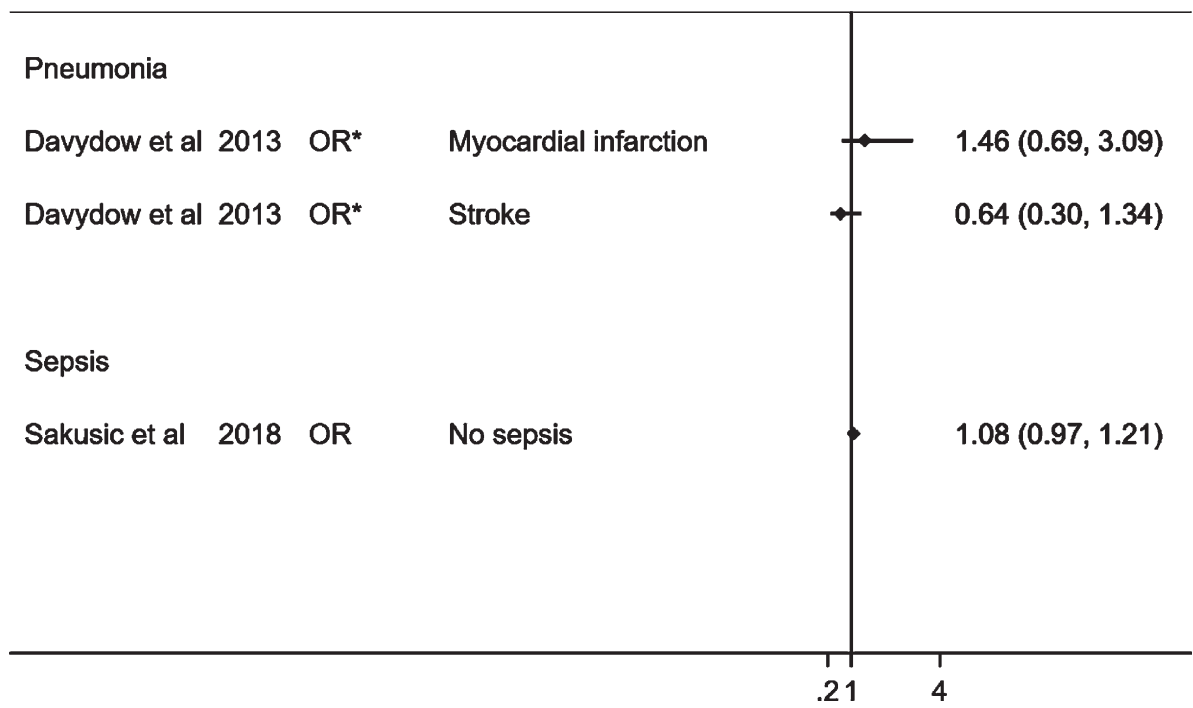

Fig. 3. Forest plot showing the effect of common bacterial infections on cognitive decline. *Unadjusted effect estimates for the study by Davydow et al., 2013 [26]. The median age in years (SD) in this study for each exposure was as follows: pneumonia 77.1 (9.4), myocardial infarction, 75.5 (8.2) and stroke 77.0 (8.4). 
Of the studies included, six studies were conducted in the United States [22-27], and three in Taiwan [28-30]. Four were historical cohort studies, which used data derived from electronic health records [22, $25,28,29]$, two were prospective cohort studies [23, $26]$, one was a secondary analysis from a randomized controlled trial [24], and two were case-control studies [27, 30].

In total, seven studies investigated sepsis [22, 23, 25, 27-30], four assessed pneumonia [23-26], and only one study considered urinary tract infections and cellulitis [25]. Two studies investigated the effect of multiple infections on dementia [23, 25]. Infections were defined using ICD-9 (International Classification of Diseases, Ninth Revision) or ICD-9CM (International Classification of Diseases, Ninth Revision, Clinical Modification) codes [22-26, 29, 30]. Ascertainment of infection was unclear in two studies [27, 28]. In terms of the setting in which infections were diagnosed, all studies defined infections in secondary care, with the exception of one study which included individuals receiving care at veterans health administration facilities [25]. These facilities comprise secondary care, outpatient sites and primary care settings. Two studies reported on the association between sepsis and dementia from the same study population in the Taiwanese health insurance database. Of these studies, one reported on vascular dementia as an outcome [28], while the other study reported on all types of dementia, Alzheimer's disease, and non-Alzheimer's disease dementia [29].

Ascertainment of dementia and cognitive decline varied across studies. Four studies defined dementia using the ICD-9 or ICD-9-CM diagnostic codes [22, $25,29,30]$, while two studies used multiple validated clinical tests to diagnose dementia [23, 24]. One study did not specify the ascertainment of dementia [28]. In one study, cognitive decline was defined using a modified version of the Telephone Interview for Cognitive Status, which is a validated measurement of cognitive impairment [26]. The other study ascertained cognitive impairment through manual review of medical records, and the use of algorithms to capture terms for cognitive impairment and dementia [27].

Sample sizes were generally smaller for studies assessing cognitive decline, ranging from 1,434 to 2,401 total population, compared to 3,602 to 417,172 in dementia studies. The age at which participants were recruited ranged widely between 18 to 75 years and older, but the mean age ranged between 65.5 to 78.6 years old.
The duration of follow up differed widely. In studies assessing cognitive decline, follow-up ranged from 3 months to 9.8 years. Among studies assessing dementia, only 3 studies reported the mean or median follow time which ranged from 2.5 to 9.0 years.

\section{Effect of infections on dementia}

Seven studies assessed dementia as an outcome (Table 2, Fig. 2) [22-25, 28-30]. In all studies, infections were associated with an increased risk of all cause dementia or vascular dementia, with effect estimates ranging from HR $1.10(95 \% \mathrm{CI} ; 1.02-1.19)$ to OR 2.60 (95\% CI;1.84-3.66).

To determine whether a meta-analysis was appropriate, we calculated heterogeneity when a sufficient number of studies were available. We decided not to meta-analyze data for the associations between sepsis or pneumonia with incident dementia due to evidence of substantial heterogeneity, $\mathrm{I}^{2}=93.6 \%$, $p=<0.01$ and $\mathrm{I}^{2}=83.9 \%, p<0.01$. Due to the limited number of studies available, we could only explore geographical location and risk of bias as potential sources for heterogeneity. Heterogeneity was explored in studies assessing incident dementia following sepsis infection as shown in Supplementary Figures 1 and 2. Removing studies conducted in Taiwan reduced heterogeneity substantially $\left(\mathrm{I}^{2}=0 \%\right.$, $p=0.406$ ); however, when studies with a domain of high risk of bias were removed, heterogeneity remained high $\left(\mathrm{I}^{2}=95.6 \%, p<0.01\right)$.

\section{Subgroup analyses}

All three studies from Taiwan reported data on subgroup analyses for age, sex, and dementia subtype as shown in Supplementary Figures 3-5. Of these, two studies reported on the association of sepsis on dementia subtype [28, 29]. In one study, sepsis was associated with an increased risk of all types of dementia 2.09 (95\% CI; 1.92-2.28) and nonAlzheimer dementias 2.20 (95\% CI; 2.01-2.41) [29]. However, the association of sepsis and Alzheimer's disease HR 1.15 (95\% CI; 0.83-1.60) was not statistically significant. In the other study, individuals with sepsis had an increased risk of vascular dementia HR 2.26 (95\% CI; 2.07-2.47) [28]. In sub-group analysis we also explored whether the effect of infections on dementia differed by sex [29, 30]. Findings from these studies showed the association of sepsis on dementia was greater in men compared to women. 
Table 3

Risk of bias summary assessments for individual domains

\begin{tabular}{|c|c|c|c|c|c|c|c|c|}
\hline $\begin{array}{ll} & \text { Key } \\
\text { O } & \text { Low risk } \\
& \text { Moderate risk } \\
\text { O } & \text { High risk } \\
& \text { Unclear risk }\end{array}$ & 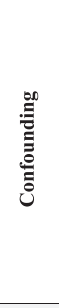 & 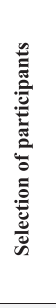 & 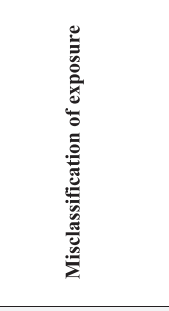 & 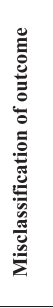 & 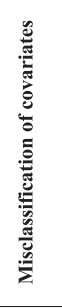 & 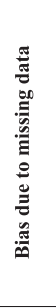 & 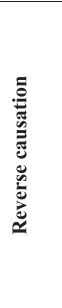 & 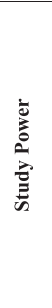 \\
\hline \multicolumn{9}{|l|}{ First author, publication year } \\
\hline Shah et al., 2013 [23] & 0 & 0 & 0 & 0 & 0 & 0 & 0 & 0 \\
\hline Guerra et al., 2012 [22] & 0 & 0 & 0 & 0 & 0 & 0 & 0 & 0 \\
\hline Mawanda et al., 2016 [25] & 0 & 0 & 0 & 0 & 0 & 0 & 0 & 0 \\
\hline Chou et al., 2017 [29] & 0 & 0 & 0 & 0 & 0 & 0 & 0 & 0 \\
\hline Chou et al., 2018 [28] & 0 & 0 & 0 & 0 & 0 & 0 & 0 & 0 \\
\hline Tate et al., 2014 [24] & 0 & 0 & 0 & 0 & 0 & 0 & 0 & 0 \\
\hline Kao et al., 2015 [30] & 0 & 0 & $\overbrace{\text { Cognitive decline }}$ & 0 & 0 & 0 & 0 & 0 \\
\hline Davydow et al., 2013 [26] & 0 & 0 & 0 & 0 & 0 & 0 & 0 & 0 \\
\hline Sakusic et al., 2018 [27] & 0 & 0 & 0 & 0 & 0 & 0 & 0 & 0 \\
\hline
\end{tabular}

Only one study investigated the effect of age on infections and dementia [30]. Kao et al. found that compared with individuals aged 45 to 64 , participants aged 65 and older showed a lower risk of dementia following sepsis HR 1.80 (95\% CI; 1.65-1.97) than those aged under 45 HR 7.32 (95\% CI; 1.85-28.9). However, these results are difficult to interpret due to the small number of events in the under 45 years age group $(n=11)$ compared to the 65 years and older group $(n=2492)$. This study also investigated the effect of sepsis severity on dementia and found that dementia incidence increased with mild HR 1.20 (95\% CI; 1.06-1.37), moderate HR 3.37 (95\% CI; 3.02-3.76) and severe HR 5.04 (95\% CI; 3.98-6.37) sepsis severity. Another study also reported an increasing trend for developing dementia with increasing sepsis severity [28].

\section{Effect of infections on cognitive decline}

Two studies assessed cognitive decline as an outcome: one following hospitalization with pneumonia and the other following admission to an intensive care unit with sepsis (Table 2, Fig. 3) [26, 27]. In one study, the effect of pneumonia hospitalization on moderate/severe cognitive impairment was compared to individuals hospitalized with stroke OR 0.64 (95\% CI; 0.3-1.34) and to those with myocardial infarction OR 1.46 (95\% CI; 0.69-3.09) [26]. In the other study, there was no association between sepsis and cognitive decline OR 1.08 (95\% CI; 0.97-1.21) in adjusted estimates [27]. Given that the definition of infections was inconsistent in these studies, we could not pool the results into a combined effect estimate and perform a meta-analysis.

\section{Risk of bias}

Our classifications and justifications for the risk of bias assessments are presented in Supplementary Table 1 and summarized in Table 3. Overall, none of the studies were classified as at low risk of bias across all domains. All studies assessing cognitive decline and three looking at dementia outcomes were considered at high risk of bias for study power. Studies assessing cognitive decline had particularly small sample sizes and wide confidence intervals compared to the dementia studies. The majority of studies assessing dementia scored a low risk of bias for confounding as these studies adjusted for age, sex, and other important covariates. Three studies did not have any domains at high risk of bias [22, 25, 29]. These studies all investigated the effect of sepsis on dementia, with hazard ratios ranging from 1.39 (95\% CI; 1.16-1.66) to 2.09 (95\% CI; 1.92-2.28). All studies were given a low rating for reverse causality as all outcomes were assessed after infection; however, three studies reported a relatively short follow up period from infection to dementia diagnosis $[22,24,30]$. Given that dementia has a long pre-clinical phase, it is thus unclear whether follow up time was long enough for dementia to develop. Further, none of the studies 
reported sufficient information on loss of follow up or how missing data were accounted for.

\section{Study quality}

The overall evidence on the associations of sepsis or pneumonia with dementia were classified as of very low quality using the GRADE assessment tool. This is because these studies were rated "serious" or "very serious" for risk of bias, inconsistency, imprecision, and indirectness (Supplementary Table 2). We did not assess the overall quality of evidence for the association of other infections on dementia or cognitive decline as only a single study was available for each exposure and outcome.

\section{DISCUSSION}

Our comprehensive systematic review identified 9 longitudinal studies examining the association of common bacterial infections with incident dementia or cognitive decline. Although a meta-analysis was not performed due to the heterogeneity of the studies, evidence from all seven studies assessing dementia found a positive association following infection with sepsis, pneumonia, urinary tract infections, or cellulitis. This association remained consistent in studies with no domains at high risk of bias. However, the overall quality of evidence was rated very low for these studies due to risk of bias, consistency, imprecision, and indirectness. Of the two studies assessing the effect of pneumonia or sepsis on cognitive decline, a lack of association was observed. However, these studies had a number of important methodological limitations including a lack of power or poor comparability, limiting the ability to draw accurate conclusions from the findings.

\section{Heterogeneity}

The high heterogeneity observed between the studies assessing incident dementia precluded a meta-analysis, despite the studies being homogenous in terms of exposure, outcome, and study design. A major source of heterogeneity may have been the differences in the country in which the study was conducted. Studies assessing the effect of sepsis on dementia were either from the United States or Taiwan, with studies from Taiwan reporting much greater effect estimates compared to those from the United States. When studies from Taiwan were removed from the meta-analysis, heterogeneity reduced substantially. However, due to the small number of studies available, we were unable to quantitatively explore heterogeneity in studies from Taiwan. Studies assessing pneumonia were all conducted in the United States, but also had high heterogeneity, however, the paucity of studies limited the ability to quantitatively explore sources of the heterogeneity.

There are a number of possible explanations for the substantial heterogeneity observed. Varying assessments were used to diagnose dementia including neuropsychological tests, ICD-9 or ICD-9-CM codes, and magnetic resonance imaging. Studies using electronic health records rely on routine medical diagnoses which can result in misclassification given that dementia is frequently under-diagnosed in these databases [31, 32]. However, further evidence suggests that recording of dementia diagnoses is changing over time, with improvements observed in more recent years $[33,34]$. Another potential issue arising from routine healthcare data is that individuals with illnesses encounter health services more frequently compared to healthy people, which could increase the likelihood of getting a dementia diagnosis. This may, however, be more likely to occur among those with chronic illnesses requiring ongoing management than with acute infections.

Another source of heterogeneity could have come from differences in the adjustment of confounders, given that the study that adjusted for a wide range of confounders including demographics, psychiatric and medical comorbidity reported weaker effect estimates in comparison to the other studies [25]. Additionally, differences in the age at recruitment and mean age of the study populations may also account for the heterogeneity. This is of importance as the risk of developing infections increases with age [35] and in turn older adults have a greater chance of developing dementia, with the risk doubling every 5 years after the age of 65 [36]. Additionally, sex representation, which ranged from $44 \%$ to $97 \%$ for men, may have contributed to heterogeneity. In our subgroup analyses, we observed differences in sex in the Taiwanese studies looking at the association between sepsis and dementia, with men at a greater risk of dementia compared to women [29,30]. Studies from Europe and the United States suggest that there is gender variation in the reduction of age-specific dementia, with some reporting a greater decline in men $[3,6,9]$ and others in women $[5,8]$.

One study did not find an association between sepsis and Alzheimer's disease. The study suggested a 
reason for this may be due to the low prevalence for a causative pathogen of Alzheimer's disease, $B$. burgdorferi, in Taiwan [29]. Another reason could be the potential to misdiagnose Alzheimer's disease. A systematic review investigating the validation of dementia cases in routine health care data from Europe, North America, and Australia found that positive predictive values of Alzheimer's disease ranged from $57 \%$ to $100 \%$ [37].

\section{Cognitive decline}

Evidence for any association of common bacterial infections with cognitive decline was limited. The study by Davydow et al. faced a number of limitations. First, there was no adjustment for confounders in this study [26]. Second, individuals hospitalized with pneumonia were compared to those with stroke and myocardial infarction. This raises issues on the suitability of these comparator groups as stroke and myocardial infarction are both risk factors for dementia and may increase the risk of pneumonia [38, 39]. The authors stated that these analyses were based on a hypothetical population, and as such the results may not be generalizable to a particular group of people. Taken together, these limitations make it difficult to extrapolate any meaningful conclusions from these results.

\section{Comparison with previous studies}

Although the association between common bacterial infections and cognitive decline was unclear, evidence from previous longitudinal studies suggests that individuals with bacterial infections are associated with worsening cognitive impairment [16, 40], with one study showing a decline in the mean score of cognitive ability, assessed using the Danish intelligence test, following hospitalization with sepsis, skin, respiratory and urological infections [41]. Other studies have found a link between sepsis and specific cognitive domains after long-term follow up [42].

Regarding the link between bacterial infections and dementia, our findings are consistent with evidence from a nested-case control study using UK primary care data which suggested that episodes of infections were associated with an increased likelihood of a dementia diagnosis [43]. This study assessed the overall effect of infections on dementia, including urinary tract and skin infections, rather than the individual effect of each infection on dementia.
Because of this, the study was not eligible for inclusion.

Individual bacterial pathogens including Helicobacter pylori, Chlamydia pneumoniae, Borelia burgdorferi, and oral spirochetal Trepenoma have been linked to Alzheimer's disease, primarily in serology based and post mortem brain studies [10, 18]. Chlamydia pneumonia and spirochetes are the focus of previous reviews and have been frequently associated with Alzheimer's disease [10, 11, 44-46]. In their review, Mawanda and Wallace suggested chronic bacterial infections, such as tuberculosis, are associated with amyloid deposition, a key hallmark of Alzheimer's disease. This is further supported in a study by Emery et al., which demonstrated an increase in actinobacteria in the brains of individuals with Alzheimer's disease compared to controls [47]. However, no single microorganism has been identified as the sole pathogen responsible for Alzheimer's disease.

\section{Mechanisms}

Mechanisms underlying the association between infections and subsequent dementia are unclear [48, 49], but several plausible pathophysiological pathways have been proposed. One such potential pathway is through systemic inflammation. Infections can induce systemic inflammation through the release of pro-inflammatory mediators which can cross the blood-brain barrier and activate cytotoxic microglia. This may result in a deterioration of cognitive function and thus increasing the risk of developing dementia [10]. In support of this mechanism, evidence from a growing number of longitudinal studies suggests that markers of systematic inflammation, such as tumor necrosis factor, nitric oxide synthase, and interleukin IL- $1 \beta$, IL- 6 , and IL18 , are involved in the pathogenesis of dementia [50, 51]. Recent findings demonstrate that when sepsis is induced in animal models, it triggers systemic inflammation which leads to accumulation of amyloid- $\beta$ and cognitive dysfunction [52, 53].

Alternatively, it is also possible that the association between infections and dementia is non-causal and may be a result of the co-occurrence of agerelated pathologies. The immune system deteriorates with age, increasing incidence of infection. Conversely, the aging immune system also induces a chronic inflammatory state which leads to tissue damage and inflammatory disease and accelerates age-related diseases such as Alzheimer's disease 
$[54,55]$. Nevertheless, the present review focused only on longitudinal studies which provide evidence of appropriate temporality between infections and dementia, thus adding to the likelihood of a possible causal relationship.

\section{Strengths and limitations}

Strengths of our study include a comprehensive search using multiple databases of published and grey literature, with no restrictions on the date, language or geographical location of the studies. Our search strategy was detailed and peer reviewed. We registered and published our protocol in order to increase the transparency of our findings. Other strengths include the inclusion of longitudinal studies to minimize reverse causality, a minimum 3 month follow up period to avoid capturing short term cognitive impairment, and requiring the use of a comparator group without infections in order to provide evidence on causality.

There are several limitations to this systematic review. First, there was a small number of longitudinal studies available, particularly for cognitive decline outcomes. Second, the high heterogeneity between the studies meant that it was not feasible to perform a meta-analysis. Third, given the long pre-clinical phase of dementia and the evidence that individuals with dementia are at a greater risk of hospitalizations and common bacterial infections [56], we cannot rule out the possibility of reverse causality. Fourth, these studies did not account for past hospitalizations with infections, as such we cannot rule out the effect of previous infections on the risk of dementia. Fifth, the generalizability of these studies is of concern. All studies were conducted in the United States or Taiwan, and there were no studies from Europe or low- or middle-income countries. Further to this, infections were predominantly diagnosed in a hospital setting. These findings may thus not be representative of individuals with less severe infections that did not require hospitalization. This may have led to an underestimation of people with infections.

\section{Implications for research and practice}

The paucity of studies available highlights the need for further large scale, longitudinal studies from populations across the world.

Our sub-group analyses suggested that the severity of sepsis is associated with an increased risk of dementia. Further research on the effect of sever- ity, frequency, and timing of infections on cognitive decline and dementia is warranted. This will be important for identifying the sub-populations most at risk of dementia. In line with this rationale, previous studies have identified a dose-response relationship between hospital contacts with infection and cognitive ability [41]. Additionally, there is evidence of gender variation between infections and dementia, as such, more work is needed to explore this possible link further as it may have implications on prevention strategies in men and women.

A key drawback of the studies included was the fact that infections were predominantly diagnosed in secondary care. This is an issue as hospitalization itself has been associated with incident cognitive decline and dementia [57-59]. Hospitalized patients are at a greater risk of nosocomial infections [60], delirium [61], and functional decline [62], which may also increase the risk of dementia. Therefore, individuals hospitalized with infections may not be representative of those with infections diagnosed in primary care. In future, studies could investigate the effect of infections diagnosed in different health care settings. Additionally, the bacterial agents responsible for infections acquired in the community or in hospital settings differ, and as such it is possible that these pathogens could have differing effects on dementia, if any at all. Future longitudinal studies investigating the link between laboratory confirmed bacterial agents and dementia could shed further light on causality.

Research on infections as potential risk factors for dementia faces a number of challenges. Firstly, the etiology of dementia is multifactorial and is likely to involve an interplay of genetic, environmental, and lifestyle factors. In addition, the fact that age is the single greatest risk factor for dementia raises challenges in disentangling the pathophysiological effects of age on dementia with the independent effects of infections on dementia. Secondly, the pathophysiological processes of dementia may begin years before dementia is diagnosed, and as such it is possible that the preclinical phase of dementia may be underway before infection occurs. Future studies with a follow up time sufficient enough for dementia to develop are recommended in order to minimize the possibility of reverse causality. Moreover, given that infections may trigger delirium, it is important for future studies to ensure that individuals are followed up long enough for delirium to resolve in order to help distinguish between delirium and long-term cognitive decline. However, as delirium itself is associated with 
cognitive decline and dementia, there is a need to better understand whether the pathological processes of infections on long-term cognitive decline are independent of delirium. Third, prospective cohort studies on infections and dementia are susceptible to selection bias. Individuals with more severe infections are associated with attrition as they are more likely to experience greater morbidity and an increased risk of mortality compared to those with less severe infections. Additionally, cognitive decline and dementia are associated with attrition during follow up and drop-out due to death $[63,64]$, which may underestimate the true effect of infections on dementia. Further studies could tackle this limitation by performing an analysis of attrition to investigate whether those lost to follow up were more likely to have impaired cognitive function. Loss of follow up is minimized in routine healthcare datasets; however, one of the limitations of these datasets is that they rely on individuals seeking health care services. As a result, health seeking behavior could affect the likelihood of a dementia diagnosis. Future studies should consider accounting for health-seeking behavior in their design or analysis.

This review suggests that infections may be involved in the development of dementia. These findings could have clinical implications in the early recognition and treatment of infections, particularly in the older population who are more susceptible to infections and are at a greater risk of dementia. Additionally, other implications include the need for strategies to improve infection control and to identify sub-populations at risk of infections and dementia.

\section{Conclusions}

Our systematic review suggests that sepsis, pneumonia, urinary tract infections, and cellulitis may be associated with an increased risk of dementia, after adjustment for multiple confounders. However, due to the paucity of longitudinal studies, further evidence from high quality studies is needed to confirm this association. Given that evidence on cognitive decline was limited by a lack of studies and small sample sizes, further large scale, well-powered studies are needed to investigate the effect of infections on cognitive decline. Infections are well-recognized to trigger delirium, as such, it is important for future work to distinguish whether the potential association between infections and cognitive decline is independent of delirium. Common bacterial infections frequently occur in the elderly, who are at an increased risk of dementia, and thus a better understanding of their role in dementia development could inform dementia risk reduction strategies.

\section{ACKNOWLEDGMENTS}

Rutendo Muzambi is supported by an Alzheimer's Society PhD studentship 379 (AS-PhD-17-013). We would like to thank the lay volunteers assigned to Rutendo's PhD studentship for their input and advice. Charlotte Warren-Gash is supported by a Wellcome Intermediate Clinical Fellowship, Wellcome Trust (201440_Z_16_Z) and Krishnan Bhaskaran holds a Sir Henry Dale Fellowship jointly funded by the Wellcome Trust and the Royal Society.

Authors' disclosures available online (https:// www.j-alz.com/manuscript-disclosures/20-0303r2).

\section{SUPPLEMENTARY MATERIAL}

The supplementary material is available in the electronic version of this article: https://dx.doi.org/10. 3233/JAD-200303.

\section{REFERENCES}

[1] Patterson C (2018) World Alzheimer Report 2018. The state of the art of dementia research: New frontiers. Alzheimer's Disease International, London, UK.

[2] World Health Organization, Dementia: A public health priority, http://www.who.int/mental_health/publications/ dementia_report_2012/en/, Accessed 06/02/19.

[3] Matthews FE, Stephan BCM, Robinson L, Jagger C, Barnes LE, Arthur A, Brayne C, Cognitive Function and Ageing Studies (CFAS) Collaboration (2016) A two decade dementia incidence comparison from the Cognitive Function and Ageing Studies I and II. Nat Commun 7, 11398.

[4] Schrijvers EM, Verhaaren BF, Koudstaal PJ, Hofman A, Ikram MA, Breteler MM (2012) Is dementia incidence declining?: Trends in dementia incidence since 1990 in the Rotterdam Study. Neurology 78, 1456-1463.

[5] Grasset L, Brayne C, Joly P, Jacqmin-Gadda H, Peres K, Foubert-Samier A, Dartigues JF, Helmer C (2016) Trends in dementia incidence: Evolution over a 10-year period in France. Alzheimers Dement 12, 272-280.

[6] Qiu C, von Strauss E, Backman L, Winblad B, Fratiglioni L (2013) Twenty-year changes in dementia occurrence suggest decreasing incidence in central Stockholm, Sweden. Neurology 80, 1888-1894.

[7] van Bussel EF, Richard E, Arts DL, Nooyens ACJ, Coloma PM, de Waal MWM, van den Akker M, Biermans MCJ, Nielen MMJ, van Boven K, Smeets H, Matthews FE, Brayne C, Busschers WB, van Gool WA, Moll van Charante EP (2017) Dementia incidence trend over 1992-2014 in the Netherlands: Analysis of primary care data. PLoS Med 14, e1002235. 
[8] Satizabal C, Beiser AS, Seshadri S (2016) Incidence of dementia over three decades in the Framingham Heart Study. N Engl J Med 375, 93-94.

[9] Rocca WA, Petersen RC, Knopman DS, Hebert LE, Evans DA, Hall KS, Gao S, Unverzagt FW, Langa KM, Larson EB, White LR (2011) Trends in the incidence and prevalence of Alzheimer's disease, dementia, and cognitive impairment in the United States. Alzheimers Dement 7, 80-93.

[10] Sochocka M, Zwolińska K, Leszek J (2017) The infectious etiology of Alzheimer's disease. Curr Neuropharmacol 15, 996-1009.

[11] Maheshwari P, Eslick GD (2015) Bacterial infection and Alzheimer's disease: A meta-analysis. J Alzheimers Dis 43, 957-966.

[12] Ebersoldt M, Sharshar T, Annane D (2007) Sepsisassociated delirium. Intensive Care Med 33, 941-950.

[13] Girard TD, Jackson JC, Pandharipande PP, Pun BT, Thompson JL, Shintani AK, Gordon SM, Canonico AE, Dittus RS, Bernard GR, Ely EW (2010) Delirium as a predictor of longterm cognitive impairment in survivors of critical illness. Crit Care Med 38, 1513-1520.

[14] Davis DH, Muniz Terrera G, Keage H, Rahkonen T, Oinas M, Matthews FE, Cunningham C, Polvikoski T, Sulkava R, MacLullich AM, Brayne C (2012) Delirium is a strong risk factor for dementia in the oldest-old: A population-based cohort study. Brain 135, 2809-2816.

[15] MacLullich AM, Beaglehole A, Hall RJ, Meagher DJ (2009) Delirium and long-term cognitive impairment. Int Rev Psychiatry 21, 30-42.

[16] Iwashyna TJ, Ely EW, Smith DM, Langa KM (2010) Longterm cognitive impairment and functional disability among survivors of severe sepsis. JAMA 304, 1787-1794.

[17] Annane D, Sharshar T (2015) Cognitive decline after sepsis. Lancet Respir Med 3, 61-69.

[18] Mawanda F, Wallace R (2013) Can infections cause Alzheimer's disease? Epidemiol Rev 35, 161-180.

[19] Muzambi R, Bhaskaran K, Brayne C, Smeeth L, WarrenGash C (2019) Common bacterial infections and risk of incident cognitive decline or dementia: A systematic review protocol. BMJ Open 9, e030874.

[20] Sterne JA, Hernán MA, Reeves BC, Savović J, Berkman ND, Viswanathan M, Henry D, Altman DG, Ansari MT, Boutron I, Carpenter JR, Chan A-W, Churchill R, Deeks JJ, Hróbjartsson A, Kirkham J, Jüni P, Loke YK, Pigott TD, Ramsay CR, Regidor D, Rothstein HR, Sandhu L, Santaguida PL, Schünemann HJ, Shea B, Shrier I, Tugwell P, Turner L, Valentine JC, Waddington H, Waters E, Wells GA, Whiting PF, Higgins JP (2016) ROBINS-I: A tool for assessing risk of bias in non-randomised studies of interventions. BMJ 355, i4919.

[21] Higgins JP, Altman DG, Gotzsche PC, Juni P, Moher D, Oxman AD, Savovic J, Schulz KF, Weeks L, Sterne JA (2011) The Cochrane collaboration's tool for assessing risk of bias in randomised trials. BMJ 343, d5928.

[22] Guerra C, Linde-Zwirble WT, Wunsch H (2012) Risk factors for dementia after critical illness in elderly Medicare beneficiaries. Crit Care 16, R233.

[23] Shah FA, Pike F, Alvarez K, Angus D, Newman AB, Lopez O, Tate J, Kapur V, Wilsdon A, Krishnan JA, Hansel N, Au D, Avdalovic M, Fan VS, Barr RG, Yende S (2013) Bidirectional relationship between cognitive function and pneumonia. Am J Respir Crit Care Med 188, 586-592.

[24] Tate JA, Snitz BE, Alvarez KA, Nahin RL, Weissfeld LA, Lopez O, Angus DC, Shah F, Ives DG, Fitzpatrick AL, Williamson JD, Arnold AM, DeKosky ST, Yende S (2014)
Infection hospitalization increases risk of dementia in the elderly. Crit Care Med 42, 1037-1046.

[25] Mawanda F, Wallace RB, McCoy K, Abrams TE (2016) Systemic and localized extra-central nervous system bacterial infections and the risk of dementia among US veterans: A retrospective cohort study. Alzheimers Dement (Amst) 4, 109-117.

[26] Davydow DS, Hough CL, Levine DA, Langa KM, Iwashyna TJ (2013) Functional disability, cognitive impairment, and depression after hospitalization for pneumonia. Am J Med 126, 615-624.e615.

[27] Sakusic A, Gajic O, Singh TD, O'Horo JC, Jenkins G, Wilson GA, Petersen R, Fryer JD, Kashyap R, Rabinstein AA (2018) Risk factors for persistent cognitive impairment after critical illness, nested case-control study. Crit Care Med $\mathbf{4 6}$, 1977-1984.

[28] Chou CH, Lee JT, Lin CC, Muo CH, Wen CP, Wang IK, Kao CH, Hsu CY, Tseng CH (2018) Association between septicemia and vascular dementia: A nation-wide population-based study. Int J Stroke 13, 160.

[29] Chou CH, Lee JT, Lin CC, Sung YF, Lin CC, Muo CH, Yang FC, Wen CP, Wang IK, Kao CH, Hsu CY, Tseng CH (2017) Septicemia is associated with increased risk for dementia: A population-based longitudinal study. Oncotarget 8, 8430084308.

[30] Kao LT, Sheu JJ, Lin HC, Tsai MC, Chung SD (2015) Association between sepsis and dementia. J Clin Neurosci 22, 1430-1433.

[31] Bradford A, Kunik ME, Schulz P, Williams SP, Singh H (2009) Missed and delayed diagnosis of dementia in primary care: Prevalence and contributing factors. Alzheimer Dis Assoc Disord 23, 306-314.

[32] Connolly A, Gaehl E, Martin H, Morris J, Purandare N (2011) Underdiagnosis of dementia in primary care: Variations in the observed prevalence and comparisons to the expected prevalence. Aging Ment Health 15, 978-984.

[33] Perera G, Stewart R, Higginson IJ, Sleeman KE (2016) Reporting of clinically diagnosed dementia on death certificates: Retrospective cohort study. Age Ageing 45, 668-673.

[34] Donegan K, Fox N, Black N, Livingston G, Banerjee S, Burns A (2017) Trends in diagnosis and treatment for people with dementia in the UK from 2005 to 2015: A longitudinal retrospective cohort study. Lancet Public Health 2, e149e156.

[35] Castle SC (2000) Clinical relevance of age-related immune dysfunction. Clin Infect Dis 31, 578-585.

[36] Jorm AF, Jolley D (1998) The incidence of dementia: A meta-analysis. Neurology 51, 728-733.

[37] Wilkinson T, Ly A, Schnier C, Rannikmäe K, Bush K, Brayne C, Quinn TJ, Sudlow CLM (2018) Identifying dementia cases with routinely collected health data: A systematic review. Alzheimers Dement 14, 1038-1051.

[38] Grau AJ, Urbanek C, Palm F (2010) Common infections and the risk of stroke. Nat Rev Neurol 6, 681-694.

[39] Warren-Gash C, Hayward AC, Hemingway H, Denaxas S, Thomas SL, Timmis AD, Whitaker H, Smeeth L (2012) Influenza infection and risk of acute myocardial infarction in England and Wales: A CALIBER self-controlled case series study. J Infect Dis 206, 1652-1659.

[40] Merli M, Lucidi C, Pentassuglio I, Giannelli V, Giusto M, Di Gregorio V, Pasquale C, Nardelli S, Lattanzi B, Venditti M, Riggio O (2013) Increased risk of cognitive impairment in cirrhotic patients with bacterial infections. J Hepatol 59, 243-250. 
[41] Benros ME, Sørensen HJ, Nielsen PR, Nordentoft M, Mortensen PB, Petersen L (2015) The association between infections and general cognitive ability in young men - a nationwide study. PLoS One 10, e0124005.

[42] Semmler A, Widmann CN, Okulla T, Urbach H, Kaiser M, Widman G, Mormann F, Weide J, Fliessbach K, Hoeft A, Jessen F, Putensen C, Heneka MT (2013) Persistent cognitive impairment, hippocampal atrophy and EEG changes in sepsis survivors. J Neurol Neurosurg Psychiatry 84, 62-69.

[43] Dunn N, Mullee M, Perry VH, Holmes C (2005) Association between dementia and infectious disease: Evidence from a case-control study. Alzheimer Dis Assoc Disord 19, 91-94.

[44] Shima K, Kuhlenbaumer G, Rupp J (2010) Chlamydia pneumoniae infection and Alzheimer's disease: A connection to remember? Med Microbiol Immunol 199, 283-289.

[45] Honjo K, van Reekum R, Verhoeff NP (2009) Alzheimer's disease and infection: Do infectious agents contribute to progression of Alzheimer's disease? Alzheimers Dement 5, 348-360.

[46] Miklossy J (2011) Alzheimer's disease - a neurospirochetosis. Analysis of the evidence following Koch's and Hill's criteria. J Neuroinflammation $\mathbf{8}, 90$.

[47] Emery DC, Shoemark DK, Batstone TE, Waterfall CM, Coghill JA, Cerajewska TL, Davies M, West NX, Allen SJ (2017) 16S rRNA next generation sequencing analysis shows bacteria in Alzheimer's post-mortem brain. Front Aging Neurosci 9, 195.

[48] Fulop T, Witkowski JM, Bourgade K, Khalil A, Zerif E, Larbi A, Hirokawa K, Pawelec G, Bocti C, Lacombe G, Dupuis G, Frost EH (2018) Can an infection hypothesis explain the beta amyloid hypothesis of Alzheimer's disease? Front Aging Neurosci 10, 224.

[49] Itzhaki RF, Lathe R, Balin BJ, Ball MJ, Bearer EL, Braak H, Bullido MJ, Carter C, Clerici M, Cosby SL, Del Tredici K, Field H, Fulop T, Grassi C, Griffin WS, Haas J, Hudson AP, Kamer AR, Kell DB, Licastro F, Letenneur L, Lovheim H, Mancuso R, Miklossy J, Otth C, Palamara AT, Perry G, Preston C, Pretorius E, Strandberg T, Tabet N, TaylorRobinson SD, Whittum-Hudson JA (2016) Microbes and Alzheimer's disease. J Alzheimers Dis 51, 979-984.

[50] Sundelof J, Kilander L, Helmersson J, Larsson A, Ronnemaa E, Degerman-Gunnarsson M, Basun H, Lannfelt L, Basu S (2009) Systemic inflammation and the risk of Alzheimer's disease and dementia: A prospective population-based study. J Alzheimers Dis 18, 79-87.

[51] Kim JW, Stewart R, Kang HJ, Bae KY, Kim SW, Shin IS, Yoon JS, Kim JM (2018) Longitudinal associations between serum cytokine levels and dementia. Front Psychiatry 9, 606.

[52] Gasparotto J, Girardi CS, Somensi N, Ribeiro CT, Moreira JCF, Michels M, Sonai B, Rocha M, Steckert AV, Barichello T, Quevedo J, Dal-Pizzol F, Gelain DP (2018) Receptor for advanced glycation end products mediates sepsis-triggered amyloid- $\beta$ accumulation, Tau phosphorylation, and cognitive impairment. J Biol Chem 293, 226-244.

[53] Wang L-M, Wu Q, Kirk RA, Horn KP, Ebada Salem AH, Hoffman JM, Yap JT, Sonnen JA, Towner RA, Bozza FA, Rodrigues RS, Morton KA (2018) Lipopolysaccharide endotoxemia induces amyloid- $\beta$ and $\mathrm{p}$-tau formation in the rat brain. Am J Nucl Med Mol Imaging 8, 86-99.

[54] Weyand CM, Goronzy JJ (2016) Aging of the immune system. Mechanisms and therapeutic targets. Ann Am Thorac Soc 13(Suppl 5), S422-S428.

[55] Martorana A, Bulati M, Buffa S, Pellicanò M, Caruso C, Candore G, Colonna-Romano G (2012) Immunosenescence, inflammation and Alzheimer's disease. Longev Healthspan 1, 8.

[56] Phelan EA, Borson S, Grothaus L, Balch S, Larson EB (2012) Association of incident dementia with hospitalizations. JAMA 307, 165-172.

[57] Ehlenbach WJ, Hough CL, Crane PK, Haneuse SJ, Carson SS, Curtis JR, Larson EB (2010) Association between acute care and critical illness hospitalization and cognitive function in older adults. JAMA 303, 763-770.

[58] Mathews SB, Arnold SE, Epperson CN (2014) Hospitalization and cognitive decline: Can the nature of the relationship be deciphered? Am J Geriatr Psychiatry 22, 465-480.

[59] Chen CC, Chiu MJ, Chen SP, Cheng CM, Huang GH (2011) Patterns of cognitive change in elderly patients during and 6 months after hospitalisation: A prospective cohort study. Int J Nurs Stud 48, 338-346.

[60] Klevens RM, Edwards JR, Richards CL Jr, Horan TC, Gaynes RP, Pollock DA, Cardo DM (2007) Estimating health care-associated infections and deaths in U.S. hospitals, 2002. Public Health Rep 122, 160-166.

[61] Siddiqi N, House AO, Holmes JD (2006) Occurrence and outcome of delirium in medical in-patients: A systematic literature review. Age Ageing 35, 350-364.

[62] Zisberg A, Shadmi E, Gur-Yaish N, Tonkikh O, Sinoff G (2015) Hospital-associated functional decline: The role of hospitalization processes beyond individual risk factors. $J$ Am Geriatr Soc 63, 55-62.

[63] Chatfield MD, Brayne CE, Matthews FE (2005) A systematic literature review of attrition between waves in longitudinal studies in the elderly shows a consistent pattern of dropout between differing studies. J Clin Epidemiol 58, 13-19.

[64] Matthews FE, Chatfield M, Freeman C, McCracken C, Brayne C, Cfas MRC (2004) Attrition and bias in the MRC cognitive function and ageing study: An epidemiological investigation. BMC Public Health 4, 12. 